\title{
The permeability barrier of nuclear pore complexes appears to operate via hydrophobic exclusion
}

\author{
Katharina Ribbeck and Dirk Görlich ${ }^{1}$ \\ ZMBH, INF 282, D-69120 Heidelberg, Germany \\ ${ }^{1}$ Corresponding author \\ e-mail: dg@zmbh.uni-heidelberg.de
}

\begin{abstract}
Nuclear pore complexes (NPCs) restrict the nucleocytoplasmic flux of most macromolecules, but permit facilitated passage of nuclear transport receptors and their cargo complexes. We found that a simple hydrophobic interaction column can mimic the selectivity of NPCs surprisingly well and that nuclear transport receptors appear to be the most hydrophobic soluble proteins. This suggests that surface hydrophobicity represents a major sorting criterion of NPCs. The rate of NPC passage of cargo-receptor complexes is, however, not dominated just by properties of the receptors. We found that large cargo domains drastically hinder NPC passage and require more than one receptor molecule for rapid translocation. This argues against a rigid translocation channel and instead suggests that NPC passage involves a partitioning of the entire translocating species into a hydrophobic phase, whereby the receptor:cargo ratio determines the solubility in that permeability barrier. Finally, we show that interfering with hydrophobic interactions causes a reversible collapse of the permeability barrier of NPCs, which is consistent with the assumption that the barrier is formed by phenylalanine-rich nucleoporin repeats that attract each other through hydrophobic interactions.

Keywords: importin/kinetics/NTF2/nuclear pore complex/ nuclear transport
\end{abstract}

\section{Introduction}

Transport of macromolecules between nucleus and cytoplasm is a fundamental and essential activity in eukaryotic cells. It proceeds through nuclear pore complexes (NPCs), which are embedded into the two membranes of the nuclear envelope (NE; Vasu and Forbes, 2001). Vertebrate NPCs have a mass of $125 \mathrm{MDa}$ and contain 30-50 different proteins, which are referred to as nucleoporins. Each nucleoporin occurs in at least eight copies per pore, adding up to a total of $\sim 1000$ individual polypeptides per NPC. NPCs function as highly selective gates and allow passage of material in two modes: passive diffusion and facilitated translocation (reviewed in Mattaj and Englmeier, 1998; Görlich and Kutay, 1999; Nakielny and Dreyfuss, 1999; Rabut and Ellenberg, 2001). Passive diffusion is fast for metabolites and small macromolecules, but becomes increasingly inefficient as the substrate approaches a size limit of 20-40 kDa (Bonner, 1978). In contrast, facilitated translocation can accommodate the rapid transport of even very large objects (for review see Daneholt, 1997; Mattaj and Englmeier, 1998; Görlich and Kutay, 1999; Nakielny and Dreyfuss, 1999; Conti and Izaurralde, 2001; Reed and Magni, 2001). It is often coupled to an input of metabolic energy, which allows transport against a gradient of chemical activity (active transport).

Facilitated translocation requires specific interactions between the translocating species and NPC components (Bayliss et al., 1999, 2000; Strasser et al., 2000; Fribourg et al., 2001; Ribbeck and Görlich, 2001). It is therefore a highly selective process and available only for 'privileged' objects with 'translocation-promoting' properties. These properties are normally conferred by nuclear transport receptors. Molecules that lack translocation-promoting properties will here be referred to as 'inert' objects. Their NPC passage is normally restricted by a permeability barrier. They can cross NPCs only in a facilitated manner when bound to a suitable nuclear transport receptor.

Importin $\beta(\operatorname{Imp} \beta)$-type transport receptors account for the majority of the nuclear transport pathways. They occur in two forms, import mediators (importins) and exportins (Mattaj and Englmeier, 1998; Weis, 1998; Wozniak et al., 1998; Görlich and Kutay, 1999; Nakielny and Dreyfuss, 1999). They circulate between nucleus and cytoplasm, recognize cargo molecules and transfer them from one side of the NE to the other. Substrate loading and release is guided by a concentration gradient of RanGTP across the NE, which is sensed through the RanGTP binding domains present in the transport receptors. A high nuclear RanGTP concentration favours cargo loading onto exportins and substrate displacement from importins, while cytoplasmic conditions with low levels of RanGTP release substrates from exportins but allow importin-cargo complexes to form (Rexach and Blobel, 1995; Fornerod et al., 1997; Kutay et al., 1997a). Importins and exportins constantly export Ran from the nucleus. Continued transport cycles therefore require a replenishment of the nuclear RanGTP pool, which is accomplished by NTF2-mediated import of RanGDP (Ribbeck et al., 1998; Smith et al., 1998), followed by RCC1-mediated nucleotide exchange to RanGTP (Bischoff and Ponstingl, 1991).

Importins and exportins can accumulate cargoes against a gradient of chemical activity, which is an energyconsuming task. Therefore, metabolic energy needs to be fed into these transport cycles. This occurs solely through the RanGTPase system (Weis et al., 1996a) and only at the level of the active regulation of cargo binding and release just mentioned. In contrast, the facilitated translocation process per se is neither active nor vectorial, but instead a fully reversible and energy-independent process (Kose et al., 1997; Nakielny and Dreyfuss, 1998; Ribbeck et al., 1998, 1999; Schwoebel et al., 1998; Englmeier et al., 1999; Nachury and Weis, 1999). 
For growing HeLa cells, it has been estimated that on average a single NPC accommodates the facilitated translocation of no less than 10-20 MDa of material per second (Ribbeck and Görlich, 2001). The maximal capacity is even higher, in the order of $80 \mathrm{MDa}$ per pore and second. Accordingly, facilitated translocation can be extremely fast and approach rates of a purely diffusioncontrolled process. The translocation channel is not unselectively open, but instead is controlled by some permeability barrier. This barrier poses little resistance to objects with optimal translocation-promoting properties, but restricts the flux of inert objects $>20 \mathrm{kDa}$ by at least two to three orders of magnitude (Bonner, 1978; Ribbeck and Görlich, 2001). The nature of the permeability barrier and the mechanism of facilitated translocation through it are central issues in the field. It is clear that the barrier must be formed by nucleoporins, and indeed the passive diffusion limit appears altered in certain nucleoporin mutants (Shulga et al., 2000).

Many nucleoporins contain phenylalanine-rich repeats (Rout and Wente, 1994), which are characterized by short clusters of hydrophobic residues (such as FxFG or GLFG) separated by very hydrophilic spacers. There are up to 30 Phe-rich repeats per nucleoporin and an estimated $10^{4}$ per NPC. Imp $\beta$-type transport receptors, NTF2 as well as mRNA export mediators bind Phe-rich repeats directly (Paschal and Gerace, 1995; Radu et al., 1995; Clarkson et al., 1996; Bachi et al., 2000; Strasser et al., 2000; Allen et al., 2001). Mutational analysis of NTF2, $\operatorname{Imp} \beta$ and the mRNA-export mediator TAP/Mex67 has demonstrated a critical role of these interactions in facilitated translocation (Bayliss et al., 1999, 2000; Strasser et al., 2000; Fribourg et al., 2001). However, the question as to how an interaction with the Phe-rich repeats can promote NPC passage is not trivial, because binding alone would cause retention of the translocating material at pores and delay its passage.

To resolve the problem, several models have been suggested. The affinity gradient model (Ben-Efraim and Gerace, 2001) explains facilitated translocation as a movement of the translocating species along binding sites of increasing affinity. A problem with this model lies in the fact that the actual NPC passage is a fully reversible process (Ribbeck et al., 1998, 1999; Englmeier et al., 1999; Nachury and Weis, 1999) and thus is facilitated equally in both directions. It is therefore not plausible that a gradient of rising affinity should be more effective than a falling one or no gradient at all. In addition, kinetic measurements indicated that high-affinity interactions are dispensable for facilitated nuclear pore passage (Ribbeck and Görlich, 2001).

The key feature of the Brownian affinity gate model (Rout et al., 2000) is an aqueous NPC channel that is surrounded by filamentous nucleoporins, which are enriched in Phe-rich binding sites for nuclear transport receptors. The central channel is assumed to be so narrow that particles above the passive diffusion limit would be unlikely to enter. The function of the repeats would be to trap and concentrate nuclear transport receptors near the channel, which should increase the probability of receptors entering the channel and diffusing through it. This model predicts that binding to the repeats is necessary and sufficient for facilitated translocation and that the trans- location rate is determined by targeting to pores. It does not explain how objects larger than the physical diameter of the narrow channel can cross the pore.

The selective phase model (Ribbeck and Görlich, 2001) assumes the central channel to be wide in diameter $(40 \mathrm{~nm})$, i.e. large enough to accommodate the largest receptor-cargo complexes known to cross NPCs (see Feldherr and Akin, 1990; Pante and Kann, 2002; discussed in Ribbeck and Görlich, 2001). The channel would not be fully open, but filled with a selective phase, formed by Phe-rich repeats, that excludes inert macromolecules but constitutes a good solvent for nuclear transport receptors. Properties of any liquid phase are determined primarily by interactions between solvent molecules, and this consideration is the key element of the model. Phe-rich repeats in the selective phase are assumed to attract each other through weak hydrophobic interactions and form a meshwork, which can restrict the flux of inert molecules that exceed the mesh size. Translocating material, however, can be incorporated into the meshwork, because it is able to interact with the Phe-rich clusters and thereby compete locally the mutual attraction between the repeats. The translocating species could thus partition selectively into the permeability barrier and use this 'selective solvation' to cross the barrier. The selective phase would seal around the translocating species and remain a barrier for inert material even when large cargoes pass.

We show here that nuclear transport receptors are collectively more hydrophobic than average cytosolic proteins, which is consistent with the assumption that they cross NPCs by partitioning into a hydrophobic phase. The selective phase model implies that not only the receptor, but also a bound cargo, becomes exposed to the permeability barrier; it thus predicts that a complete partitioning into the selective phase will not be dominated just by favourable properties of the receptor, but also counteracted by inert cargo domains. Indeed, we find that large cargoes can drastically delay NPC passage, but increasing the receptor-to-cargo ratio can compensate for this effect. Finally, we used small molecules to mildly disrupt hydrophobic interactions and observed that such treatment causes a reversible collapse of the permeability barrier of the pore, which in turn supports a key element of the selective phase model.

\section{Results and discussion}

\section{Surface hydrophobicity of soluble proteins correlates with translocation competence and appears to be the major sorting criterion of NPCs}

Mutational analyses of nuclear transport receptors strongly suggest that interactions between the translocating species and Phe-rich repeats are necessary and possibly even sufficient for facilitated NPC passage (Bayliss et al., 1999, 2000; Strasser et al., 2000; Fribourg et al., 2001; Ribbeck and Görlich, 2001). Structural studies of $\operatorname{Imp} \beta$ and the $\mathrm{TAP} / \mathrm{p} 15$ complex revealed details of such interactions and demonstrated that the hydrophobic side chains of the nucleoporin repeat intrude into hydrophobic pockets of the transport mediators (Bayliss et al., 2000; Fribourg et al., 2001).

To test whether such hydrophobic cavities represent general and obligate determinants for facilitated trans- 


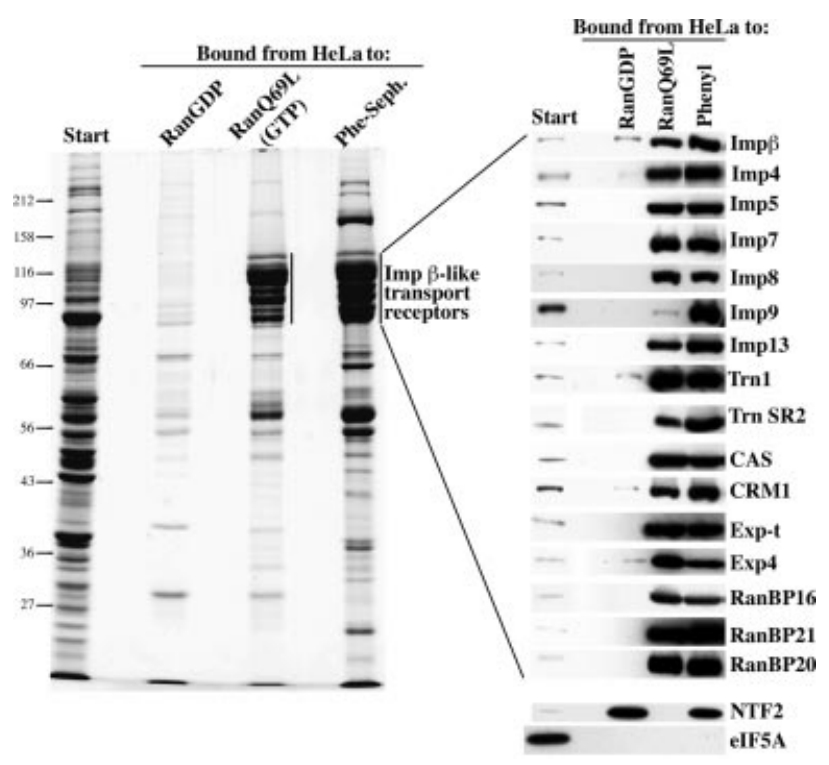

Fig. 1. Surface hydrophobicity is one marker for translocation competence. A cytosolic extract from HeLa cells was prepared and subjected to binding to immobilized RanGDP, RanGTP or phenyl-Sepharose (low substitution). Analysis of starting material and bound fractions was by SDS-PAGE followed by Coomassie Blue staining (left panel) or western blotting with antibodies raised against the indicated nuclear transport receptors (right panels). Phenyl-Sepharose retrieved $\operatorname{Imp} \beta$ transport receptors with specificity similar to that of RanGTP. Certain receptors (e.g. Imp9) were recovered with even higher efficiency by phenyl-Sepharose than by RanGTP. Likewise, NTF2 bound phenylSepharose as efficiently as its import substrate RanGDP. In contrast, typical cytosolic proteins, such as eIF-5A, did not bind to the hydrophobic matrix under these stringent conditions (see also Materials and methods and main text). Load in the bound fraction corresponds to $20 \times$ the starting material.

location, we incubated a total cytosolic extract with a hydrophobic interaction column under very stringent binding conditions, i.e. using a low substitution phenylSepharose in the absence of ammonium sulfate. The bound fraction showed a protein pattern that resembled strikingly that of nuclear transport receptors retrieved on a RanGTP column (compare corresponding lanes in Figure 1). Indeed, western blotting confirmed that all 16 members of the $\operatorname{Imp} \beta$ family tested, as well as NTF2, were highly enriched by the phenyl-Sepharose (Figure 1). The same also applied to the TAP/p15 complex (not shown), which mediates mRNA export (Grüter et al., 1998; Katahira et al., 1999). In contrast, the vast majority of cytoplasmic proteins (which are incapable of facilitated NPC passage) were not recovered with the column (see eIF5A blot for a representative example). Phenyl-Sepharose is thus, despite its extremely simple composition, a highly selective affinity matrix for factors capable of facilitated NPC passage and represents an excellent mimic for the selectivity of NPCs. The experiment demonstrates that nuclear transport receptors are distinguished from inert molecules by a greater surface hydrophobicity. It is consistent with all models, which assume that these receptors traverse NPCs by means of hydrophobic interactions with NPC components. In addition, it provides a convenient procedure to deplete selectively and quantitatively cytosolic extracts of nuclear transport receptors.

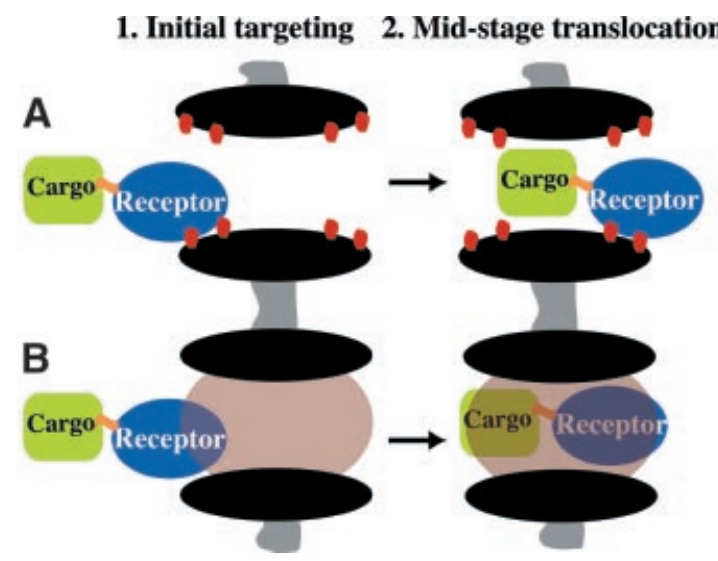

Fig. 2. Two scenarios for facilitated NPC passage. (A) The receptor contacts Phe-rich motifs (red dots) at the periphery of the channel, which triggers a movement through the otherwise empty (i.e. purely aqueous) and rigid channel. (B) In the second scenario, receptor-cargo complexes must partition completely into a tightly sealing selective phase, which implies that the receptor (which is attracted by the phase) as well as the cargo (which is repelled by the phase) become exposed to the permeability barrier.

The hydrophobicity of nuclear transport receptors must, however, also be well balanced in order to avoid aggregation, recruitment of heat shock proteins and a too-stable binding to nucleoporins, which would not only delay translocation, but also block NPCs for other transport pathways (Kutay et al., 1997b; Lane et al., 2000; Quimby et al., 2001).

\section{Cargo domains cause a 'drag' that slows down receptor-mediated NPC passage}

The special surface properties of nuclear transport receptors permit facilitated NPC passage, while inert molecules are normally retained by the permeability barrier. However, inert macromolecules can also cross NPCs in a facilitated manner, provided they recruit an appropriate transport receptor. The resulting receptorcargo complexes have composite surfaces, with elements of the receptor and those areas of the cargo that are not covered by the receptor. This poses the critical question as to what extent a cargo can affect the NPC passage of the receptor. The magnitude of such a 'cargo-effect' could potentially be used to explore properties of the permeability barrier (see Figure 2 for a scheme).

If the rate of NPC passage were determined solely by the on-rate of targeting to NPCs, and NPC passage proceeded thereafter through a fully open and empty channel, then the cargo effect on the translocation rate would be small. A larger cargo would only decrease the diffusion coefficient of the receptor/ cargo complex as the mass of this complex increases. In this scenario, $\operatorname{Imp} \beta$ dependent import of a $90 \mathrm{kDa}$ cargo would be only $10 \%$ slower than the import of a $40 \mathrm{kDa}$ substrate. In contrast, if the movement proceeded through a tightly sealing permeability barrier, into which the entire translocating species must dissolve, then the cargo domain should have a major impact on the partition coefficient and thereby exert a significant inhibitory effect on the translocation rate.

To resolve the issue, we first compared NTF2-mediated import of Ran that was fused to a his tag $(1.7 \mathrm{kDa})$, to 
24 seconds NTF2-mediated import of:

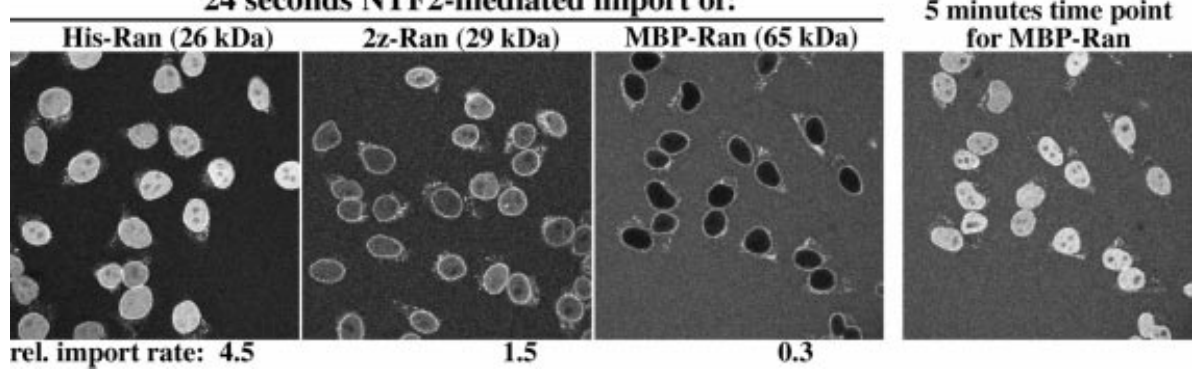

Fig. 3. Inert cargo domains hinder NPC passage of Ran. Ran was expressed with three different N-terminal tags in order to obtain Ran derivatives of increasing size. These tags were a his tag, a zz domain (IgG binding domain from protein A) or MBP. Nuclear import of the Alexa 488-labelled fusion proteins $(1 \mu \mathrm{M}$ each) was performed in the presence of NTF2 (one homodimer per Ran molecule) and an energy regenerating system (see Materials and methods). The nucleocytoplasmic Ran distribution was recorded in real time by confocal scans through the import mixtures. Quantitation of the data revealed that influx of zzRan and MBP-Ran occurred 3-fold and 15-fold, respectively, more slowly than influx of his-tagged Ran. The 5 min time point is a control to demonstrate that MBP-Ran is capable of nuclear accumulation even though its influx is slow.

A 9 seconds Importin $\beta$-mediated import of:

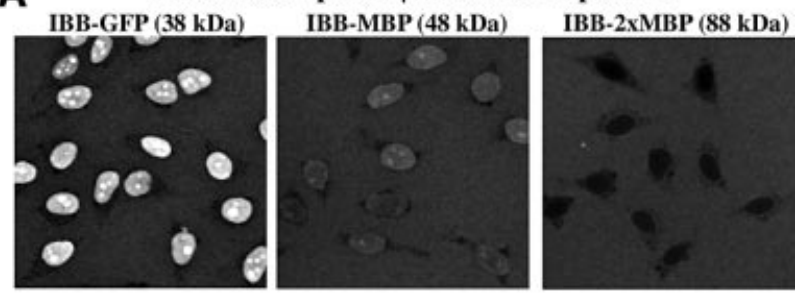

B Enlarged section of IBB-2xMBP sample

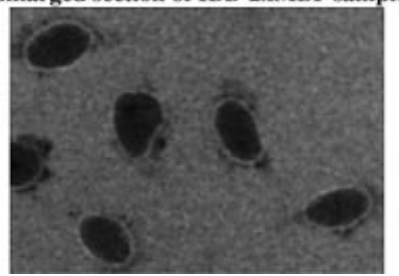

Fig. 4. (A) The rate of facilitated translocation is determined not only by properties of the receptor, but also by those of the cargo. Three different inert proteins, namely GFP, MBP and an MBP dimer $(2 \times$ MBP $)$, were each fused to an IBB domain (a potent $\operatorname{Imp} \beta$-dependent import signal). The GFP fusion was detected through its intrinsic fluorescence. Fluorescent MBP fusion proteins had been labelled with Alexa 488 maleimide. The fluorescent fusion proteins $(0.5 \mu \mathrm{M}$ final $)$ were pre-bound to stoichiometric amounts of $\operatorname{Imp} \beta$ and their import into nuclei of permeabilized cells was allowed in the presence of Ran and an energy-regenerating system. The distribution of the fusions was determined by confocal fluorescence microscopy. Panels show the reactions after $9 \mathrm{~s}$ of import. Even though the three substrates were linked to identical import signals and imported under identical conditions by the same receptor, their import rates were strikingly different. Thus, the translocation rate is not only determined by the receptor and the type of signal, but also by the 'rest' of the cargo domain. (B) Enlarged field of the IBB- $2 \times$ MBP sample from (A). Clear NPC staining is evident even against the high background of cytoplasmic substrate.

a zz domain $(14 \mathrm{kDa})$ or to the maltose binding protein MBP (40 kDa). The fusions interfere neither with the Ran-NTF2 interaction (not shown) nor with targeting of the Ran-NTF2 complexes to NPCs (see Figure 3). However, the zz domain slows down NPC passage of Ran 3-fold and the MPB domain even 15-fold as compared with the his-tagged Ran. Thus, inert cargo domains can drastically hinder NPC passage.

To test whether that conclusion also applies to other transport pathways, we next studied $\operatorname{Imp} \beta$-dependent cargo import. For this purpose, we fused a potent $\operatorname{Imp} \beta$ dependent import signal, the IBB domain (Görlich et al., 1996; Weis et al., 1996b) to three differently sized cargoes (Figure 4A). These were the green fluorescent protein (GFP; $30 \mathrm{kDa}), \mathrm{MBP}(40 \mathrm{kDa})$ and two copies of MBP $(2 \times$ MBP; $80 \mathrm{kDa})$. Stoichiometric complexes with $\operatorname{Imp} \beta$ were pre-formed and imported in the presence of Ran and energy. Figure 4A compares the influx rates of the three fusion proteins and reveals the striking differences. After $9 \mathrm{~s}$, the IBB-GFP fusion $(38 \mathrm{kDa}$ ) had already reached a 5 -fold nuclear accumulation over the cytoplasmic concentration. In contrast, the influx of IBB-MBP (48 kDa) turned out to be three times slower and that of IBB- $2 \times$ MBP $(88 \mathrm{kDa})$ even 20 -fold slower. It should be noted, however, that the IBB $-2 \times$ MBP substrate gave a typical NPC-like rim staining even against the high background of cytoplasmic substrate (see Figure 4B), indicating that the initial targeting to NPCs was efficient, but the subsequent transfer through the NPCs was very slow.

\section{Large cargo domains require more than one receptor molecule for efficient NPC passage}

Is the NPC passage of large objects then necessarily slow or is it the receptor:cargo ratio that matters? Cytoplasmic injections into Xenopus oocytes demonstrated previously a faster nuclear import of nucleoplasmin with five signals than with fewer signals (Dingwall et al., 1982). This would be consistent with the assumption that the rate of NPC passage increases with the number of bound receptor molecules. However, it does not prove the point. More signals make binding of a limiting receptor more probable, and that alone would sufficiently explain the greater import rate, even if the nucleoplasmin complexes with a single receptor molecule or with multiple receptors traverse NPCs at identical rates.

To resolve the issue, the NPC passage of defined receptor-cargo complexes must be measured. A technical problem is to obtain homogeneous populations of receptor-cargo complexes. If the importin were added at 1:1 stoichiometry to a substrate with two identical import signals, then a statistical mix of cargo with none, one and two receptors would form. This can only be avoided by providing the cargo with two distinct signals that are specific for different importins. We therefore fused the 
54 seconds import of IBB-2xMBP-M3 with Ran, energy, and:

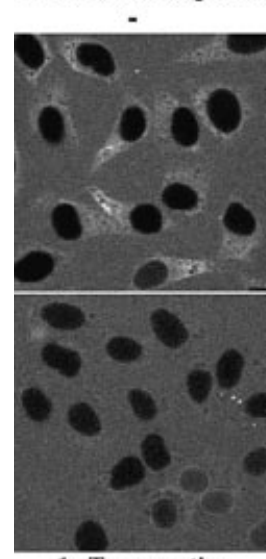

1x Transportin

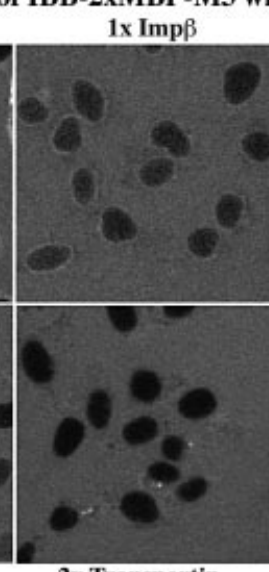

2x Transportin

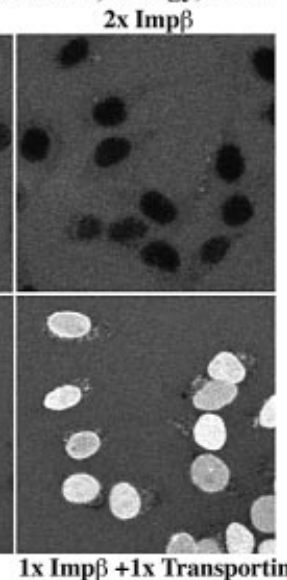

$1 x \operatorname{Imp} \beta+1 x$ Transportin
Fig. 5. Large cargoes can cross NPCs rapidly, provided that more than one receptor is recruited. The fluorescent import substrate $(0.4 \mu \mathrm{M})$ contains an $\mathrm{N}$-terminal IBB domain for Imp $\beta$-dependent import, a double MBP cargo segment and a C-terminal M3 domain for transportin-mediated import. Import was performed with Ran, an energyregenerating system and the indicated combinations of transportin and $\operatorname{Imp} \beta$. Receptor:cargo stoichiometry is indicated. As the permeabilized cells contain significant amounts of endogenous transportin and $\operatorname{Imp} \beta$, endogenous receptors had to be quenched with either $2 \mu \mathrm{M}$ unlabelled MBP-M3 (minus transportin samples) or IBB-MBP (minus Imp $\beta$ samples). Transportin and Imp $\beta$ show a high degree of cooperativity in the import of this fusion protein. Relative influx rates were as follows: 0.28 with $\operatorname{Imp} \beta, 0.18$ with transportin and 3.2 with $\operatorname{Imp} \beta$ plus transportin. The $2 \times \operatorname{Imp} \beta$ and $2 \times$ transportin control reactions verified that the added transportin or $\operatorname{Imp} \beta$ was not limiting for the import reactions and that further increase in their concentration did not improve import.

$2 \times$ MBP cargo segment with an N-terminal IBB domain for Imp $\beta$-mediated import and a C-terminal M3 domain, which is a transportin-specific import signal (Pollard et al., 1996). With either Imp $\beta$ or transportin alone, the fusion protein eventually accumulated inside nuclei (not shown), but the influx was very slow (Figure 5) and was also not further stimulated by increasing the concentration of the single receptor (Figure 5, see $2 \times \operatorname{Imp} \beta$ and $2 \times$ transportin samples). In contrast, import was rapid when both $\operatorname{Imp} \beta$ and transportin had been pre-bound to the substrate, demonstrating that import of large cargoes can indeed be fast, provided the receptor:cargo ratio is sufficiently high (Figure 5).

The data argue against a key element of the Brownian affinity gate model (Rout et al., 2000), in which Phe-rich repeats facilitate NPC passage only by feeding substrates into a rigid, narrow central channel. Even though the affinity gate model (or other models that assume a rigid channel) would also predict that large cargoes hinder the receptor passage as the size of cargo-receptor complexes approaches or even exceeds the diameter of the channel, such a model cannot explain why recruiting a second receptor would make the passage so rapid again (Figure 4).

If the rate of NPC passage were in the first instance determined by the on-rate of targeting to NPCs, then the degree of cooperativity between transportin and $\operatorname{Imp} \beta$ should be, at the most, an additive one. With $\operatorname{Imp} \beta$ or transportin alone, relative influx rates of 0.28 and 0.18 , respectively, were observed, the sum of both being 0.46 . However, we observed upon the combination of $\operatorname{Imp} \beta$ plus transportin a much faster relative influx of 3.2. This rules out the possibility that targeting to NPCs alone is rate limiting. Instead, it supports the assumption that the limiting step of facilitated translocation is a partitioning of the entire translocating species into a tightly sealing permeability barrier, whereby the receptor:cargo ratio determines the partition coefficient or 'solubility' in the phase (see Figure 2B).

The drag against NPC passage should depend not only on a cargo's size but also on its hydrophilicity. In particular, the exposure of excessive charges should delay translocation. One can now argue that typical importins and import adaptors possess highly acidic domains yet are capable of rapid NPC passage. We do not see this as a contradiction of the conclusion just drawn. First, translocation-inhibitory elements can be compensated for by translocation-promoting elements elsewhere in the translocating species (that is why a cargo traverses NPCs so much more quickly when bound to a transport receptor). Secondly, these receptors are certainly optimized to carry basic cargoes (which cover the acidic patches of the importins) and not to translocate 'empty'. In fact, we observed that cargo-free importin $\alpha$ is imported by $\operatorname{Imp} \beta$ more quickly when the negative charges of its NLS binding sites were neutralized by a short SV40 NLS peptide of complementary charge (not shown).

\section{Mild interference with hydrophobic interactions causes a non-selective opening of NPCs}

Our data indicate that NPCs contain no rigid channel, but instead a flexible permeability barrier, adjustable to the dimensions of the translocating material. This should imply that the maintenance of the permeability barrier and facilitated translocation through the barrier represent intimately linked phenomena. As hydrophobic interactions between Phe-rich repeats and nuclear transport receptors promote the facilitated translocation through NPCs, it appears reasonable to assume that hydrophobic interactions originating from Phe-rich repeats are also critical for the maintenance of the permeability barrier.

If so, then one would predict that interfering with hydrophobic interactions would increase the flux of objects whose NPC passage is normally restricted. As a probe for the permeability of NPCs towards inert molecules, we chose MBP (without import signals). Its passive diffusion through untreated pores into nuclei was rather slow, and after $48 \mathrm{~s}$ it was still largely excluded from the nuclei (Figure 6A). As a mild perturbant of hydrophobic interactions, we used trans-cyclohexane-1,2-diol, which elutes nuclear transport receptors from phenyl-Sepharose (not shown) and resembles a 'mini detergent', whose polar group is an ethylene glycol and whose apolar part is a butylene moiety. When the reagent was added along with the fluorescent substrate, MBP rapidly entered the nuclei, indicating that the permeability barrier had completely collapsed within seconds. It should be noted that the treatment not only increased the influx of MBP, but also that of other inert proteins such as IgG (not shown). However, it did not further accelerate the already rapid facilitated translocation of, for example, transportin or NTF2 (not shown).

At this point, we had to ensure that the effect was not caused by some non-selective damage, such as loss or irreversible denaturation of nucleoporins or even perfor- 
A

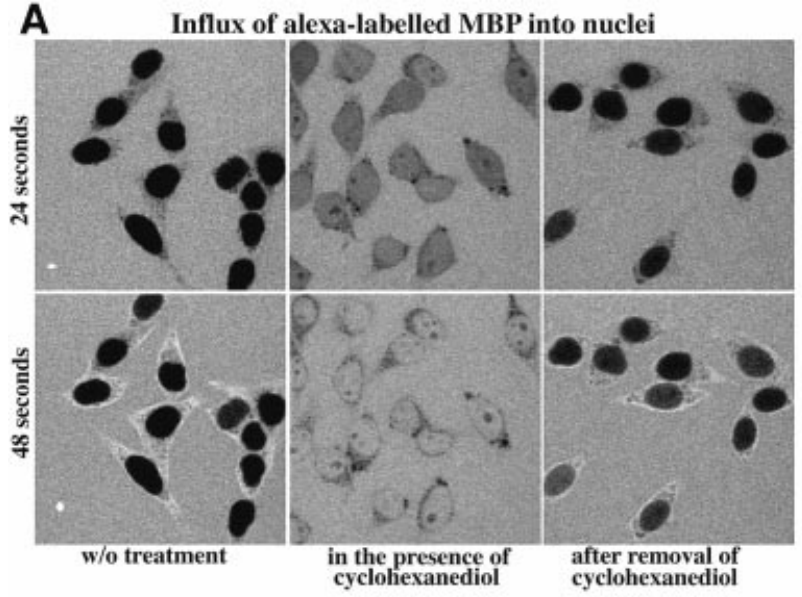

B

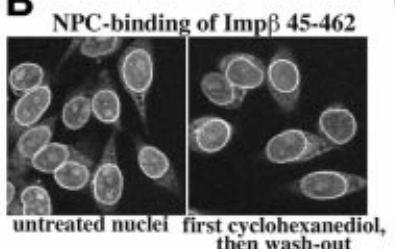

C

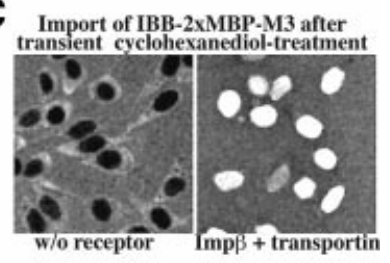

Fig. 6. Interference with hydrophobic interactions causes a reversible, non-selective opening of NPCs. (A) Left panels show influx of fluorescent MBP into nuclei without further addition after 24 and $48 \mathrm{~s}$. Middle panels: simultaneous addition of trans-cyclohexane-1,2-diol (7\% w/v) along with the substrate resulted in a greatly enhanced MBP influx. Right panels: nuclei were pre-incubated for $1 \mathrm{~min}$ with cyclohexanediol and the reagent was washed out before the influx of MBP was measured. The removal of cyclohexanediol restored the permeability barrier. (B) Comparison of NPC binding of a fluorescent $\operatorname{Imp} \beta$ 45-462 fragment either for untreated nuclei (left) or for nuclei after a 1 min cyclohexanediol treatment and subsequent removal of the reagent. (C) Nuclei were transiently treated with cyclohexanediol as in (A) and (B) and import of the IBB-2 $\times$ MBP-M3 fusion was measured. The import was strictly receptor dependent, indicating that NPCs had not been irreversibly damaged by the treatment.

ation of the NE. We therefore washed out the reagent after $1 \mathrm{~min}$ and tested thereafter the intactness of the NE and the functionality of NPCs: the permeability barrier towards MBP was indeed largely restored upon removal of the reagent (Figure 6A) and NPCs were capable of binding a fluorescent $\operatorname{Imp} \beta$ fragment (Figure 6B). However, the most stringent control was that nuclear import of the signal-containing IBB- $2 \times$ MBP-M3 fusion protein remained strictly Ran, energy and receptor dependent (Figure 6C).

Figure 7 shows that $5 \% n$-hexane-1,2-diol also caused a collapse of the permeability barrier, while $n$-hexane-1,2,3triol (which is slightly less hydrophobic) had no detectable effect. This indicates that the hydrophobicity of hexane1,2-diol was crucial in triggering the non-selective opening of the pore.

Our last control is that the NPC gating by hexane-1,2diol (Figure 7) or cyclohexanediol (not shown) could be fully abrogated by the lectin wheatgerm agglutinin (WGA). WGA is an established inhibitor of facilitated translocation (Finlay et al., 1987) and acts by binding $\mathrm{N}$-acetylglucosamine moieties that are dispersed between the Phe-rich repeats of many nucleoporins. This indicates strongly that the rapid influx of MBP was not due to (transient or irreversible) damage to the NE and instead

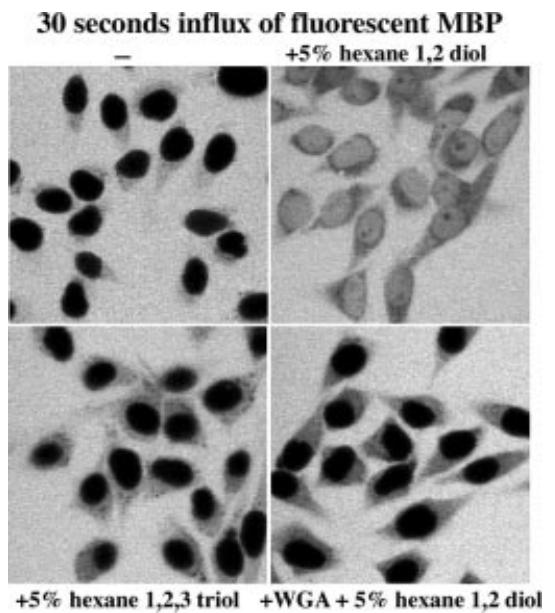

Fig. 7. The permeability barrier of NPCs is maintained by weak hydrophobic interactions. The permeability of NPCs for inert molecules was measured as the influx of fluorescent MBP into nuclei. Where indicated, 5\% hexane-1,2-diol or hexane-1,2,3-triol was added together with the substrate. Hexanediol, but not the less hydrophobic hexanetriol, caused a non-selective opening of the NPCs and allowed rapid MPB influx. This effect could be fully suppressed by pre-incubation of NPCs with $0.2 \mathrm{mg} / \mathrm{ml}$ WGA. For details, see text.

suggests that the hydrophobic compounds act directly on the Phe-rich nucleoporin repeats. Normally, WGA hardly affects passive diffusion of inert objects through NPCs. Its effect on the hexanediol-induced NPC passage is probably so striking because the tetrameric lectin cross-links adjacent Phe-rich repeats to each other and thereby prevents a hexanediol-induced rearrangement of the repeats.

\section{Conclusions}

In summary, we conclude that the maintenance of the permeability barrier and facilitated NPC passage are intimately linked phenomena. While nuclear transport receptors promote NPC passage, cargo domains can drastically counteract the process. This is most plausibly explained if not only the receptors, but also the cargoes become directly exposed to a permeability barrier that is of hydrophobic nature and tightly seals around the translocating species. This barrier is apparently based on hydrophobic exclusion of hydrophilic material and collapses as the hydrophobic effect is weakened. Nuclear transport receptors overcome this exclusion, because they are more hydrophobic than average soluble proteins and interact directly with the hydrophobic Phe-rich cluster of the nucleoporin repeats. These repeats are also ideal candidates to form the building blocks of the barrier and our model implies that the barrier is maintained not only by the mere presence of the repeats within the central channel, but is further tightened by (weak) hydrophobic attractions between them. For purely hydrophilic macromolecules, this would give a sieve-like structure whose mesh size determines the passive diffusion limit of the pores. Passage of larger objects requires a breaking of the inter-repeat interactions, which is energetically unfavourable for purely hydrophilic material, but favourable for nuclear transport receptors that bind the repeats and thereby compete the repeat-repeat interactions locally. The permeability barrier can then seal around the 
translocating species and maintain a barrier for inert material even when large objects pass.

\section{Materials and methods}

\section{Binding assays}

Fifteen grams of HeLa cells were lysed hypotonically. The resulting extract $(50 \mathrm{ml})$ was adjusted to $50 \mathrm{mM}$ Tris- $\mathrm{HCl}, 50 \mathrm{mM} \mathrm{NaCl}, 5 \mathrm{mM}$ magnesium acetate, $250 \mathrm{mM}$ sucrose (binding buffer conditions) and centrifuged to sediment particles $>40 \mathrm{~S}$. An $800 \mu \mathrm{l}$ aliquot of the supernatant (cytosolic extract) was rotated for $2 \mathrm{~h}$ with $40 \mu \mathrm{l}$ of the following affinity matrices: zzRanGDP wild type or zzRanQ69LGTP immobilized at $2 \mathrm{mg} / \mathrm{ml}$ to IgG Sepharose or phenyl-Sepharose low substitution (Pharmacia). Unbound material was removed and the matrices were washed five times with $1 \mathrm{ml}$ binding buffer. Elution from immobilized Ran was with $1.5 \mathrm{M}$ magnesium chloride; elution from phenyl-Sepharose was with SDS sample buffer.

\section{Antibodies}

Antibodies against NTF2, Imp $\beta$, Imp13, transportin, CAS, Imp5, Imp7, exportin 4 and exportin-t have been described previously (see Mingot et al., 2001 and references therein). Antibodies against Imp4 (Jäkel et al., 2002) were raised against the recombinant protein. Antibodies against Imp8, Imp9, transportin SR2, RanBP16, -20 and -21 were raised against peptides that correspond to the C-termini of the proteins. All antibodies were used after affinity purification on the immobilized antigens.

\section{Expression constructs}

Expression of IBB-GFP, IBB-MBP, IBB- $2 \times$ MBP and IBB- $2 \times$ MBP-M3 was from pQE60 (Qiagen) with C-terminal his tags. The IBB domain corresponding to the $65 \mathrm{~N}$-terminal residues of RchI (a human importin $\alpha$ ) was fused in front of the cargo domains. MBP was amplified by PCR with appropriate restriction sites from pMal-c2 (NEB). The M3 domain, corresponding to residues 256-320 of hnRNP A1, was fused to the C-terminus of the cargo domain. The MBP constructs contained two engineered cysteines for attachment of the fluorophore. The expression constructs for N-his $\operatorname{Imp} \beta, \mathrm{C}$-his transportin and the constituents of the Ran mix were described previously (see Ribbeck and Görlich, 2001 and references therein).

\section{Recombinant protein expression and purification}

Expression of zzRan wt and zzRanQ69L has been described previously. GDP- and GTP-bound forms were separated on SP-Sepharose FF. MBP-Ran was expressed and purified the same way. N-his $\operatorname{Imp} \beta$, C-his transportin, IBB-GFP, IBB-MBP, IBB- $2 \times$ MBP and IBB $-2 \times$ MBP-M3 were purified on Ni-NTA-agarose followed by gel filtration on Superdex 200. Buffers for MBP-derived constructs contained $5 \mathrm{mM}$ maltose. Fluorescence labelling of MBP fusions was performed with stoichiometric amounts of Alexa 488 maleimide (Molecular Probes).

\section{Import assays}

All nuclear import reactions into nuclei of permeabilized cells (Adam et al., 1990) were performed on coverslips and recorded directly on the non-fixed samples using time-lapse confocal laser scanning microscopy as described previously (Ribbeck and Görlich, 2001). Nuclear import of Ran was performed into nuclei preloaded with transportin (Ribbeck et al., 1998). Import of IBB and M3 fusion proteins was in the presence of a Ran mix, consisting of $3 \mu \mathrm{M}$ Ran, $0.6 \mu \mathrm{M}$ NTF2 (monomers), $0.2 \mu \mathrm{M}$ RanBP1 and $0.1 \mu \mathrm{M}$ Rna1p. The energy-regenerating system consists of $10 \mathrm{mM}$ creatine phosphate, $50 \mu \mathrm{g} / \mathrm{ml}$ creatine kinase, $0.5 \mathrm{mM}$ GTP and $0.5 \mathrm{mM}$ ATP. The import buffer was $20 \mathrm{mM}$ HEPES-KOH $\mathrm{pH} 7.5$, $110 \mathrm{mM}$ potassium acetate, $5 \mathrm{mM}$ magnesium acetate, $0.5 \mathrm{mM}$ EGTA, $250 \mathrm{mM}$ sucrose.

\section{Acknowledgements}

We wish to thank Petra Rübmann and Uschi Jäkle for excellent technical help, J.Ellenberg, M.Pool and T.Rapoport for critical reading of the manuscript, U.Kutay for the IBB-GFP and the MBP-Ran constructs, and the DFG for financial support.

\section{References}

Adam,S.A., Marr,R.S. and Gerace,L. (1990) Nuclear protein import in permeabilized mammalian cells requires soluble cytoplasmic factors. J. Cell Biol., 111, 807-816.
Allen,N.P., Huang,L., Burlingame,A. and Rexach,M. (2001) Proteomic analysis of nucleoporin interacting proteins. J. Biol. Chem., 276, 29268-29274.

Bachi,A. et al. (2000) The C-terminal domain of TAP interacts with the nuclear pore complex and promotes export of specific CTE-bearing RNA substrates. RNA, 6, 136-158.

Bayliss,R., Ribbeck,K., Akin,D., Kent,H.M., Feldherr,C.M., Görlich,D. and Stewart,M. (1999) Interaction between NTF2 and xFxFGcontaining nucleoporins is required to mediate nuclear import of RanGDP. J. Mol. Biol., 293, 579-593.

Bayliss,R., Littlewood,T. and Stewart,M. (2000) Structural basis for the interaction between FxFG nucleoporin repeats and importin- $\beta$ in nuclear trafficking. Cell, 102, 99-108.

Ben-Efraim,I. and Gerace,L. (2001) Gradient of increasing affinity of importin $\beta$ for nucleoporins along the pathway of nuclear import. J. Cell Biol., 152, 411-417.

Bischoff,F.R. and Ponstingl,H. (1991) Catalysis of guanine nucleotide exchange on Ran by the mitotic regulator RCC1. Nature, 354, 80-82.

Bonner,W.M. (1978) Protein migration and accumulation in nuclei. In Busch,H. (ed.), The Cell Nucleus. Vol. 6, part C. Academic Press, New York, NY, pp. 97-148.

Clarkson,W.D., Kent,H.M. and Stewart,M. (1996) Separate binding sites on nuclear transport factor 2 (NTF2) for GDP-Ran and the phenylalanine-rich repeat regions of nucleoporins p62 and Nsp1p. J. Mol. Biol., 263, 517-524.

Conti,E. and Izaurralde,E. (2001) Nucleocytoplasmic transport enters the atomic age. Curr. Opin. Cell Biol., 13, 310-319.

Daneholt,B. (1997) A look at messenger RNP moving through the nuclear pore. Cell, 88, 585-588.

Dingwall,C., Sharnick,S.V. and Laskey,R.A. (1982) A polypeptide domain that specifies migration of nucleoplasmin into the nucleus. Cell, 30, 449-458.

Englmeier,L., Olivo,J.C. and Mattaj,I.W. (1999) Receptor-mediated substrate translocation through the nuclear pore complex without nucleotide triphosphate hydrolysis. Curr. Biol., 9, 30-41.

Feldherr,C.M. and Akin,D. (1990) The permeability of the nuclear envelope in dividing and nondividing cell cultures. J. Cell Biol., 111, $1-8$.

Finlay,D.R., Newmeyer,D.D., Price,T.M. and Forbes,D.J. (1987) Inhibition of in vitro nuclear transport by a lectin that binds to nuclear pores. J. Cell Biol., 104, 189-200.

Fornerod,M., Ohno,M., Yoshida,M. and Mattaj,I.W. (1997) Crm1 is an export receptor for leucine rich nuclear export signals. Cell, 90, 1051-1060.

Fribourg,S., Braun,I.C., Izaurralde,E. and Conti,E. (2001) Structural basis for the recognition of a nucleoporin FG repeat by the NTF2-like domain of the TAP/p15 mRNA nuclear export factor. Mol. Cell, 8 , 645-656.

Görlich,D. and Kutay,U. (1999) Transport between the cell nucleus and the cytoplasm. Annu. Rev. Cell Dev. Biol., 15, 607-660.

Görlich,D., Henklein,P., Laskey,R.A. and Hartmann,E. (1996) A 41 amino acid motif in importin $\alpha$ confers binding to importin $\beta$ and hence transit into the nucleus. EMBO J., 15, 1810-1817.

Grüter,P., Tabernero,C., von Kobbe,C., Schmitt,C., Saavedra,C., Bachi,A., Wilm,M., Felber,B.K. and Izaurralde,E. (1998) TAP, the human homolog of Mex67p, mediates CTE-dependent RNA export from the nucleus. Mol. Cell, 1, 649-659.

Jäkel,S., Mingot,J.M., Schwarzmaier,P., Hartmann,E. and Görlich,D. (2002) Importins fulfil a dual function as nuclear import receptors and cytoplasmic chaperones for exposed basic domains. EMBO J., 21, 377-386.

Katahira,J., Strasser,K., Podtelejnikov,A., Mann,M., Jung,J.U. and Hurt,E. (1999) The Mex67p-mediated nuclear mRNA export pathway is conserved from yeast to human. EMBO J., 18, 2593-2609.

Kose,S., Imamoto,N., Tachibana,T., Shimamoto,T. and Yoneda,Y. (1997) Ran-unassisted nuclear migration of a $97-\mathrm{kD}$ component of nuclear pore-targeting complex. J. Cell Biol., 139, 841-849.

Kutay,U., Bischoff,F.R., Kostka,S., Kraft,R. and Görlich,D. (1997a) Export of importin $\alpha$ from the nucleus is mediated by a specific nuclear transport factor. Cell, 90, 1061-1071.

Kutay,U., Izaurralde,E., Bischoff,F.R., Mattaj,I.W. and Görlich,D. (1997b) Dominant-negative mutants of importin- $\beta$ block multiple pathways of import and export through the nuclear pore complex. EMBO J., 16, 1153-1163.

Lane,C.M., Cushman,I. and Moore,M.S. (2000) Selective disruption of nuclear import by a functional mutant nuclear transport carrier. J. Cell Biol., 151, 321-332. 
Mattaj,I.W. and Englmeier,L. (1998) Nucleocytoplasmic transport: the soluble phase. Апnи. Rev. Biochem., 67, 265-306.

Mingot,J.M., Kostka,S., Kraft,R., Hartmann,E. and Görlich,D. (2001) Importin 13: a novel mediator of nuclear import and export. EMBO J., 20, 3685-3694.

Nachury,M.V. and Weis,K. (1999) The direction of transport through the nuclear pore can be inverted. Proc. Natl Acad. Sci. USA, 96, 9622-9627.

Nakielny,S. and Dreyfuss,G. (1998) Import and export of the nuclear protein import receptor transportin by a mechanism independent of GTP hydrolysis. Curr. Biol., 8, 89-95.

Nakielny,S. and Dreyfuss,G. (1999) Transport of proteins and RNAs in and out of the nucleus. Cell, 99, 677-690.

Pante,N. and Kann,M. (2002) Nuclear pore complex is able to transport macromolecules with diameters of $\sim 39 \mathrm{~nm}$. Mol. Biol. Cell, 13, 425-434.

Paschal,B.M. and Gerace,L. (1995) Identification of NTF2, a cytosolic factor for nuclear import that interacts with nuclear pore protein $\mathrm{p} 62$. J. Cell Biol., 129, 925-937.

Pollard,V.W., Michael,W.M., Nakielny,S., Siomi,M.C., Wang,F. and Dreyfuss,G. (1996) A novel receptor-mediated nuclear protein import pathway. Cell, 86, 985-994.

Quimby,B.B., Leung,S.W., Bayliss,R., Harreman,M.T., Thirumala,G., Stewart,M. and Corbett,A.H. (2001) Functional analysis of the hydrophobic patch on nuclear transport factor 2 involved in interactions with the nuclear pore in vivo. J. Biol. Chem., 276, 38820-38829.

Rabut,G. and Ellenberg,J. (2001) Nucleocytoplasmic transport: diffusion channel or phase transition. Curr. Biol., 11, R551-R554.

Radu,A., Moore,M.S. and Blobel,G. (1995) The peptide repeat domain of nucleoporin Nup98 functions as a docking site in transport across the nuclear pore complex. Cell, 81, 215-222.

Reed,R. and Magni,K. (2001) A new view of mRNA export: separating the wheat from the chaff. Nature Cell Biol., 3, E201-E204.

Rexach,M. and Blobel,G. (1995) Protein import into nuclei: association and dissociation reactions involving transport substrate, transport factors and nucleoporins. Cell, 83, 683-692.

Ribbeck,K. and Görlich,D. (2001) Kinetic analysis of translocation through nuclear pore complexes. EMBO J., 20, 1320-1330.

Ribbeck,K., Lipowsky,G., Kent,H.M., Stewart,M. and Görlich,D. (1998) NTF2 mediates nuclear import of Ran. EMBO J., 17, 6587-6598.

Ribbeck,K., Kutay,U., Paraskeva,E. and Görlich,D. (1999) The translocation of transportin-cargo complexes through nuclear pores is independent of both Ran and energy. Curr. Biol., 9, 47-50.

Rout,M.P. and Wente,S.R. (1994) Pores for thought: nuclear pore complex proteins. Trends Cell Biol., 4, 357-364.

Rout,M.P., Aitchison,J.D., Suprapto,A., Hjertaas,K., Zhao,Y. and Chait,B.T. (2000) The yeast nuclear pore complex: composition, architecture and transport mechanism. J. Cell Biol., 148, 635-651.

Schwoebel,E.D., Talcott,B., Cushman,I. and Moore,M.S. (1998) Randependent signal-mediated nuclear import does not require GTP hydrolysis by Ran. J. Biol. Chem., 273, 35170-35175.

Shulga,N., Mosammaparast,N., Wozniak,R. and Goldfarb,D.S. (2000) Yeast nucleoporins involved in passive nuclear envelope permeability. J. Cell Biol., 149, 1027-1038.

Smith,A., Brownawell,A. and Macara,I.G. (1998) Nuclear import of ran is mediated by the transport factor NTF2. Curr. Biol., 8, 1403-1406.

Strasser,K., Bassler,J. and Hurt,E. (2000) Binding of the Mex67p/Mtr2p heterodimer to FXFG, GLFG and FG repeat nucleoporins is essential for nuclear mRNA export. J. Cell Biol., 150, 695-706.

Vasu,S.K. and Forbes,D.J. (2001) Nuclear pores and nuclear assembly. Curr. Opin. Cell Biol., 13, 363-375.

Weis,K. (1998) Importins and exportins: how to get in and out of the nucleus. Trends Biochem. Sci., 23, 185-189.

Weis,K., Dingwall,C. and Lamond,A.I. (1996a) Characterization of the nuclear protein import mechanism using Ran mutants with altered nucleotide binding specificities. EMBO J., 15, 7120-7128.

Weis,K., Ryder,U. and Lamond,A.I. (1996b) The conserved aminoterminal domain of hSRP1 $\alpha$ is essential for nuclear protein import. EMBO J., 15, 1818-1825.

Wozniak,R.W., Rout,M.P. and Aitchison,J.D. (1998) Karyopherins and kissing cousins. Trends Cell Biol., 8, 184-188.

Received January 21, 2002; revised March 27, 2002; accepted April 3, 2002 